\title{
Optimal control of diffusion coefficients via decoupling fields
}

\author{
Stefan Ankirchner*1 and Alexander Fromm ${ }^{\dagger 1}$ \\ ${ }^{1}$ Institute for Mathematics, University of Jena, Ernst-Abbe-Platz 2, 07743 Jena, Germany
}

October 11, 2017

\begin{abstract}
We consider a diffusion control problem, where the controller totally determines the state's diffusion coefficient but has no influence on the state's drift rate. By using the Pontryagin maximum principle we characterize an optimal control in terms of the adjoint forward-backward stochastic differential equation (FBSDE), turning out to be fully coupled. We use the method of decoupling fields for proving that the adjoint FBSDE possesses a solution.
\end{abstract}

2010 Mathematics Subject Classification. 93E20, 60H30, 49J55.

Keywords. Optimal stochastic control, diffusion coefficient, forward-backward stochastic differential equation, decoupling field.

\section{Introduction}

Let $\left(M_{t}^{\alpha}\right)$ be a stochastic process with controlled dynamics of the form $d M_{t}^{\alpha}=\mu\left(t, M_{t}^{\alpha}\right) d t+$ $\alpha_{t} d W_{t}$, where $\mu$ is affine linear in its second argument and $W$ is a one-dimensional Brownian motion. In this article we consider the control problem that consists in minimizing, among a suitable class of controls $\alpha$, the target functional

$$
E\left[\int_{0}^{T} f\left(t, M_{t}^{\alpha}, \alpha_{t}\right) d t+g\left(M_{T}^{\alpha}\right)\right]
$$

where $f$ and $g$ are nice functions, in particular convex in $M$ and $\alpha$.

This control problem arises in situations where one can control a state's fluctuation intensity but not its drift, and where one aims at steering the state into a target area. To give an explicit example, $M^{\alpha}$ may describe the position of a particle in a medium with temperature $\alpha$. By heating or cooling the medium the particle's fluctuations increase or decrease respectively. The function $f$ reflects the costs involved by any temperature change.

Diffusion control problems arise also in portfolio optimization. In this context $M^{\alpha}$ can be interpreted as a portfolio value process with volatility $\alpha$. A reduction of the portfolio's

\footnotetext{
*s.ankirchner@uni-jena.de

†alexander.fromm@uni-jena.de
} 
volatility involves hedging costs $f$. The function $-g$ can be taken to be a utility function. The optimal control of diffusion coefficients appear also in other fields of applications, see e.g. [16] for examples arising in biology.

Assuming a Markovian framework, one can choose a Hamilton-Jacobi-Bellman (HJB) approach and characterize the value function in terms of the HJB equation. In a non-Markovian framework the problem of minimizing (1) seems to be unsolved, to the best of our knowledge.

Our solution method is based on the maximum principle, leading to a probabilistic representation of the value function's sensitivity w.r.t. the controlled space variable. More precisely, we reduce the control problem to an adjoint forward-backward stochastic differential equation (FBSDE). The adjoint FBSDE turns out to be fully coupled, and hence it is a priori not clear whether a solution exists. Our idea is to use the so-called method of decoupling fields for proving that the fully coupled system possesses indeed a solution.

In order to make our idea work, we first transform the adjoint FBSDE so as to reduce the dependence of the forward diffusion coefficient on the control. The decoupling field of the transformed FBSDE can be controlled by using the convexity properties of the cost functions. More precisely, we show that the space derivative of the decoupling field is bounded and bounded away from zero. This allows to conclude existence of a solution of the adjoint equation and hence to obtain an optimal control for the problem of minimizing (1). We remark that the convexity assumptions also guarantee that the maximum principle applies in the first place.

We now explain how our solution method compares to other approaches. One can strive to obtain a probabilistic representation of the value function itself (and not only of its derivative). In the Markovian case the value function satisfies a fully non-linear PDE, allowing a representation in terms of a 2nd order BSDE (see [21]). Our approach focuses on the derivative of the value function, which satisfies a semi-linear PDE and hence has a representation in terms of a standard FBSDE.

A difficulty of the HJB approach arises from the fact that the diffusion coefficient is unbounded in the control. In order to circumvent this difficulty, [4] introduce a tailor-made modification of the viscosity solution concept. The control problem considered in [4] is more general than the problem of the present article. In contrast to [4], our approach does not require that the function $g$ has strictly smaller growth in $x$ than the function $f$ in $a$.

Finally, a further advantage of our method compared to the HJB approach is that it allows for a non-Markovian setting, i.e. the functions $f$ and $g$ can additionally depend, in a progressively measurable way, on the Brownian paths.

The literature provides many examples of problems involving the optimal control of a diffusion coefficient. We do not strive to give an overview on this classical type of control problem, but select some articles that seem closest to the problem we study in the current article. A simple problem version with infinite time horizon is discussed in Example 7.6, [20]. Specific problems with a finite time horizon have been studied in [19], [9] and [10]. A reverse diffusion control problem is solved by McNamara [15]: he determines the reward functions for which a given bang-bang diffusion control is optimal. A diffusion control problem within an exponential martingale model is studied in [2].

As mentioned earlier, our approach is rooted in reducing the initial control problem to an FBSDE. It is a longstanding challenge to find conditions guaranteeing that a given fully coupled FBSDE possesses a solution. Sufficient conditions are provided e.g. in [11], [17], [14], [18], [5], [12] (see also references therein). The method of decoupling fields, developped in [6] (see also the precursor articles [13], [7] and [12]), is practically useful for determining whether a solution exists. A decoupling field describes the functional dependence of the 
backward part $Y$ on the forward component $X$. If the coefficients of a fully coupled FBSDE satisfy a Lipschitz condition, then there exists a maximal non-vanishing interval possessing a solution triplet $(X, Y, Z)$ and a decoupling field with nice regularity properties. The method of decoupling fields consists in analyzing the dynamics of the decoupling field's gradient in order to determine whether the FBSDE has a solution on the whole time interval $[0, T]$. The method can be successfully applied to various problems involving coupled FBSDE: In [8] solutions to a quadratic strongly coupled FBSDE with a two-dimensional forward equation are constructed to obtain solutions to the Skorokhod embedding problem for Gaussian processes with nonlinear drift. In Chapter 5 of [6] the problem of utility maximization in incomplete markets is treated for a general class of utility functions via construction of solutions to the associated coupled FBSDE. In the more recent work [3], the method is used to obtain solutions to the problem of optimal position targeting for general cost functionals.

The paper is organized as follows. In Section 1 we rigorously describe the problem and its mathematical set-up. In Section 2 we apply the maximum principle to reduce the control problem to the adjoint FBSDE. Section 3 provides a brief introduction into the method of decoupling fields. In Section 4 we first transform the adjoint FBSDE so as to dampen the dependence of the forward diffusion on the control. We then prove existence of a solution by using the method of decoupling fields. In Section 5 we illustrate the construction of a solution with an explicit example. Finally, in Section 6 we explain heuristically how the adjoint FBSDE is connected to the control problem's HJB equation.

\section{Problem formulation}

Let $T>0$ be a deterministic finite time horizon. Let $W$ be a Brownian motion on a complete probability space $(\Omega, \mathcal{F}, \mathbb{P})$ and denote by $\left(\mathcal{F}_{t}\right)_{t \in[0, T]}$ the smallest filtration satisfying the usual conditions and containing the filtration generated by $W$.

Let $g: \Omega \times \mathbb{R} \rightarrow \mathbb{R}$ be measurable and $f: \Omega \times[0, T] \times \mathbb{R} \times \mathbb{R} \rightarrow \mathbb{R}$ be measurable such that for all $(m, a) \in \mathbb{R}^{2}$ the mapping $(\omega, t) \mapsto f(\omega, t, m, a)$ is progressively measurable. We make the following additional assumptions on $f$ and $g$ :

(C0) For every fixed pair $(\omega, t) \in \Omega \times[0, T]$ the mappings $(m, a) \mapsto f(t, m, a)$ and $m \mapsto g(m)$ are convex, with $f$ being strictly convex in $a$. Note that we follow the usual convention and omit the function argument $\omega$.

(C1) $g(\cdot)$ and $f(t, \cdot, \cdot)$ are twice continuously differentiable. Moreover, $g^{\prime}, f_{m}$ and $f_{a}$ are Lipschitz continuous in the last two components and satisfy

$$
\left\|\left(\left|f_{m}\right|+\left|f_{a}\right|\right)(\cdot, \cdot, 0,0)\right\|_{\infty}<\infty \text { and }\left\|g^{\prime}(0)\right\|_{\infty}<\infty .
$$

(C2) There exists a positive constant $\delta_{l}>0$ such that $f_{a a} \geq \delta_{l}$ everywhere. Throughout we assume that $\delta_{l}$ denotes the largest constant with this property.

Notice that $(\mathrm{C} 1)$ implies that there exists a $\delta_{u} \in\left[\delta_{l}, \infty\right)$ such that for all $(\omega, t, m, a) \in \Omega \times$ $[0, T] \times \mathbb{R} \times \mathbb{R}$ we have

$$
f_{a a}(t, m, a) \leq \delta_{u} .
$$

Moreover, (C1) implies that $f_{m}$ and $f_{a}$ grow at most linearly in $m$ and $a$. 
Let $\mathcal{A}$ be the set of all progressively measurable $\alpha: \Omega \times[0, T] \rightarrow \mathbb{R}$ such that $\mathbb{E}\left[\int_{0}^{T} \alpha_{s}^{2} \mathrm{~d} s\right]<$ $\infty$. For all $m \in \mathbb{R}$ and $\alpha \in \mathcal{A}$ we define

$$
M_{t}^{m, \alpha}=m+\int_{0}^{t}\left(b_{s}+B_{s} M_{s}^{m, \alpha}\right) \mathrm{d} s+\int_{0}^{t} \alpha_{s} d W_{s},
$$

where $b, B: \Omega \times[0, T] \rightarrow \mathbb{R}$ are progressively measurable and bounded processes. As outlined in the introduction, our aim is to solve the following problem:

$$
\text { Minimize } J(m, \alpha):=\mathbb{E}\left[\int_{0}^{T} f\left(s, M_{s}^{m, \alpha}, \alpha_{s}\right) d s+g\left(M_{T}^{m, \alpha}\right)\right] \text { over all } \alpha \in \mathcal{A} .
$$

For simplicity we sometimes write $M^{\alpha}$ or just $M$ instead of $M^{m, \alpha}$. In other words, for given $m$, the goal is to choose $\alpha$ from the set $\mathcal{A}$ of admissible controls in such a way that $J$ is minimized.

\section{Reducing the problem to an FBSDE}

The so-called Hamiltonian of the control problem (4) is defined by

$$
H(t, m, a, y, z):=\left(b_{t}+B_{t} m\right) y+a z+f(t, m, a),
$$

for $t \in[0, T]$ and $(m, a, z) \in \mathbb{R} \times \mathbb{R} \times \mathbb{R}$. Notice that

$$
\min _{a \in \mathbb{R}} H(t, m, a, y, z)=\left(b_{t}+B_{t} m\right) y-f^{*}(t, m,-z),
$$

where $f^{*}(t, m, \cdot)$ is the convex conjugate of $f(t, m, \cdot)$. Observe that condition $(\mathrm{C} 2)$ guarantees that $f^{*}$ assumes real values only. For the following observation we need both $(\mathrm{C} 1)$ and $(\mathrm{C} 2)$.

The minimum in (5) is attained at $a=f_{3}^{*}(t, m,-z)$, where $f_{3}^{*}$ denotes the partial derivative of $f^{*}$ with respect to the last component. This partial derivative exists since $f$ is differentiable and, by $(\mathrm{C} 2)$, the image of $f_{a}(t, m, \cdot)$ is the whole real line. More precisely, using Fermat's theorem applied to the minimization problem $\min _{a \in \mathbb{R}} H(t, m, a, y, z)=\min _{a \in \mathbb{R}} H(t, m, a, y, z)$ one can deduce

$$
a=f_{3}^{*}(t, m,-z)=f_{a}^{-1}(t, m,-z),
$$

where $f_{a}^{-1}(t, m, \cdot)$ denotes the inverse of the function $f_{a}(t, m, \cdot)$, which is strictly increasing.

The so-called adjoint forward-backward stochastic differential equation (FBSDE) for the control problem (4) is given by

$$
\begin{aligned}
M_{s}^{m} & =m+\int_{0}^{s}\left(b_{r}+B_{r} M_{r}^{m}\right) \mathrm{d} r+\int_{0}^{s} f_{3}^{*}\left(r, M_{r}^{m},-Z_{r}^{m}\right) d W_{r}, \\
Y_{s}^{m} & =g^{\prime}\left(M_{T}^{m}\right)-\int_{s}^{T} Z_{r}^{m} d W_{r}+\int_{s}^{T}\left(B_{r} Y_{r}+f_{m}\left(r, M_{r}^{m}, f_{3}^{*}\left(r, M_{r}^{m},-Z_{r}^{m}\right)\right)\right) d r
\end{aligned}
$$

for all $s \in[0, T]$. To simplify the notations, when there is no ambiguity, $\left(M^{m}, Y^{m}, Z^{m}\right)$ will be denoted by $(M, Y, Z)$. In order to rigorously define what we mean by a solution of (6) we introduce the following process spaces. For any $t_{0} \in[0, T)$ we denote by $\mathcal{H}_{t_{0}, T}^{2}$ the set of all $\left(\mathcal{F}_{t}\right)$-progressively measurable processes $\left(X_{t}\right)_{t \in\left[t_{0}, T\right]}$ such that $E \int_{t_{0}}^{T} X_{t}^{2} d t<\infty$. We define $\mathcal{H}_{T}^{2}:=\mathcal{H}_{0, T}^{2}$.

A solution to (6) is a triplet $(M, Y, Z)=\left(M^{m}, Y^{m}, Z^{m}\right)$ of progressively measurable processes such that 
- $M, Y$ and $Z$ are processes in $\mathcal{H}_{T}^{2}$,

- $M$ and $Y$ are continuous processes,

- the two equations (6) are satisfied a.s. for every fixed $s \in[t, T]$.

Constructing solutions to the above FBSDE is important for the following reason:

Proposition 2.1. If there exists a solution $(M, Y, Z)$ of (6), then an optimal control for problem (4) is given by

$$
\alpha_{s}=f_{3}^{*}\left(s, M_{s},-Z_{s}\right), \quad s \in[0, T] .
$$

Proof. We adapt the proof of Theorem 5.2 in [22] to our setting. For $m \in \mathbb{R}$ and $\bar{\alpha} \in \mathcal{A}$ let $\bar{M}=M^{m, \bar{\alpha}}$ be the associated state process. Let us define $\delta M:=\bar{M}-M$ and $\delta \alpha:=\bar{\alpha}-\alpha$. Note that $\bar{M}_{0}=M_{0}=m$, such that $\delta M_{0}=0$. Since $g$ is convex we have a.s.

$$
Y_{T} \delta M_{T}-Y_{0} \delta M_{0}=Y_{T} \delta M_{T}=g^{\prime}\left(M_{T}\right) \delta M_{T} \leq g\left(\bar{M}_{T}\right)-g\left(M_{T}\right) .
$$

At the same time Itô's formula proves that

$$
\begin{aligned}
Y_{t} \delta M_{t}-Y_{0} \delta M_{0}= & \int_{0}^{t}\left(\delta M_{s}\right) \mathrm{d} Y_{s}+\int_{0}^{t} Y_{s} \mathrm{~d}\left(\delta M_{s}\right)+\int_{0}^{t}\left(\delta \alpha_{s}\right) Z_{s} \mathrm{~d} s \\
= & -\int_{0}^{t}\left(\delta M_{s}\right)\left(B_{s} Y_{s}+f_{m}\left(s, M_{s}, \alpha_{s}\right)\right) \mathrm{d} s+\int_{0}^{t}\left(\delta M_{s}\right) Z_{s} \mathrm{~d} W_{s} \\
& +\int_{0}^{t} Y_{s} B_{s}\left(\delta M_{s}\right) \mathrm{d} s+\int_{0}^{t} Y_{s}\left(\delta \alpha_{s}\right) \mathrm{d} W_{s}+\int_{0}^{t}\left(\delta \alpha_{s}\right) Z_{s} \mathrm{~d} s \\
= & \int_{0}^{t}\left(\left(\delta \alpha_{s}\right) Z_{s}-\left(\delta M_{s}\right) f_{m}\left(s, M_{s}, \alpha_{s}\right)\right) \mathrm{d} s+ \\
& +\int_{0}^{t}\left(Y_{s}\left(\delta \alpha_{s}\right)+\left(\delta M_{s}\right) Z_{s}\right) \mathrm{d} W_{s},
\end{aligned}
$$

for all $t \in[0, T]$. Now note that

$$
H_{a}\left(s, M_{s}, \alpha_{s}, Y_{s}, Z_{s}\right)=Z_{s}+f_{a}\left(s, M_{s}, f_{3}^{*}\left(s, M_{s},-Z_{s}\right)\right)=Z_{s}-Z_{s}=0 .
$$

Together with the convexity of $H$ this implies

$$
\begin{aligned}
H\left(s, \bar{M}_{s}, \bar{\alpha}_{s}, Y_{s}, Z_{s}\right)-H\left(s, M_{s}, \alpha_{s}, Y_{s}, Z_{s}\right) \geq & H_{m}\left(s, M_{s}, \alpha_{s}, Y_{s}, Z_{s}\right)\left(\delta M_{s}\right) \\
& +H_{a}\left(s, M_{s}, \alpha_{s}, Y_{s}, Z_{s}\right)\left(\delta \alpha_{s}\right) \\
= & \left(B_{s} Y_{s}+f_{m}\left(s, M_{s}, \alpha_{s}\right)\right)\left(\delta M_{s}\right) .
\end{aligned}
$$

Thus, due to the definition of $H$

$$
\begin{aligned}
f\left(s, \bar{M}_{s}, \bar{\alpha}_{s}\right)-f\left(s, M_{s}, \alpha_{s}\right)= & H\left(s, \bar{M}_{s}, \bar{\alpha}_{s}, Y_{s}, Z_{s}\right)-H\left(s, M_{s}, \alpha_{s}, Y_{s}, Z_{s}\right) \\
& -B_{s}\left(\delta M_{s}\right) Y_{s}-Z_{s}\left(\delta \alpha_{s}\right) \\
\geq & f_{m}\left(s, M_{s}, \alpha_{s}\right)\left(\delta M_{s}\right)-Z_{s}\left(\delta \alpha_{s}\right) .
\end{aligned}
$$


Let $\tau$ be a $[0, T]$-valued stopping time such that $M, \bar{M}$ and $Y$ are bounded on $[0, \tau]$. Formula (8), together with inequality (9), implies

$$
\mathbb{E}\left[Y_{\tau} \delta M_{\tau}-Y_{0} \delta M_{0}\right] \geq \mathbb{E}\left[\int_{0}^{\tau}\left(f\left(s, M_{s}, \alpha_{s}\right)-f\left(s, \bar{M}_{s}, \bar{\alpha}_{s}\right)\right) d s\right],
$$

where all integrals are well-defined, since $M, \bar{M}, Y$ are bounded on $[0, \tau], \alpha$ and $\bar{\alpha}$ are square integrable and $f$ is at most quadratic in $a$. Note that

$$
\sup _{s \in[0, T]}\left|M_{s}\right|, \quad \sup _{s \in[0, T]}\left|\bar{M}_{s}\right| \text { and } \sup _{s \in[0, T]}\left|Y_{s}\right|
$$

are square integrable. Choosing an appropriate localizing sequence $\tau_{n} \rightarrow T$ of stopping times we can pass to the limit using dominated convergence and obtain

$$
\mathbb{E}\left[Y_{T} \delta M_{T}-Y_{0} \delta M_{0}\right] \geq \mathbb{E}\left[\int_{0}^{T}\left(f\left(s, M_{s}, \alpha_{s}\right)-f\left(s, \bar{M}_{s}, \bar{\alpha}_{s}\right)\right) d s\right] .
$$

Combining (9) with (7) we obtain

$$
\mathbb{E}\left[g\left(\bar{M}_{T}\right)-g\left(M_{T}\right)\right] \geq \mathbb{E}\left[\int_{0}^{T}\left(f\left(s, M_{s}, \alpha_{s}\right)-f\left(s, \bar{M}_{s}, \bar{\alpha}_{s}\right)\right) d s\right] .
$$

This leads to

$$
J(m, \bar{\alpha}) \geq J(m, \alpha)
$$

which shows optimality of $\alpha$.

\section{The method of decoupling fields}

As mentioned above, solving (6) is crucial in constructing optimal controls. As a key result of this paper we prove in Section 4 the solvability of (6).

Note that even under our Lipschitz assumptions, it is not trivial to show well-posedness of (6) due to its coupled nature. It is necessary to take more subtle structural properties into account to conduct the proof. Our argumentation will be based on the so-called method of decoupling fields which we will briefly sum up in this section.

For a fixed finite time horizon $T>0$, we consider a complete filtered probability space $\left(\Omega, \mathcal{F},\left(\mathcal{F}_{t}\right)_{t \in[0, T]}, \mathbb{P}\right)$, where $\mathcal{F}_{0}$ consists of all null sets, $\left(W_{t}\right)_{t \in[0, T]}$ is a 1-dimensional Brownian motion and $\mathcal{F}_{t}:=\sigma\left(\mathcal{F}_{0},\left(W_{s}\right)_{s \in[0, t]}\right)$ with $\mathcal{F}:=\mathcal{F}_{T}$. The dynamics of an FBSDE is given by

$$
\begin{aligned}
& X_{s}=X_{0}+\int_{0}^{s} \mu\left(r, X_{r}, Y_{r}, Z_{r}\right) d r+\int_{0}^{s} \sigma\left(r, X_{r}, Y_{r}, Z_{r}\right) d W_{r}, \\
& Y_{t}=\xi\left(X_{T}\right)-\int_{t}^{T} f\left(r, X_{r}, Y_{r}, Z_{r}\right) d r-\int_{t}^{T} Z_{r} d W_{r},
\end{aligned}
$$

for $s, t \in[0, T]$ and $X_{0} \in \mathbb{R}$, where $(\xi,(\mu, \sigma, f))$ are measurable functions such that

$$
\begin{array}{ll}
\xi: \Omega \times \mathbb{R} \rightarrow \mathbb{R}, & \mu:[0, T] \times \Omega \times \mathbb{R} \times \mathbb{R} \times \mathbb{R} \rightarrow \mathbb{R}, \\
\sigma:[0, T] \times \Omega \times \mathbb{R} \times \mathbb{R} \times \mathbb{R} \rightarrow \mathbb{R}, & f:[0, T] \times \Omega \times \mathbb{R} \times \mathbb{R} \times \mathbb{R} \rightarrow \mathbb{R},
\end{array}
$$


Throughout the whole section $\mu, \sigma$ and $f$ are assumed to be progressively measurable with respect to $\left(\mathcal{F}_{t}\right)_{t \in[0, T]}$.

A decoupling field comes with an even richer structure than just a classical solution $(X, Y, Z)$.

Definition 3.1. Let $t \in[0, T]$. A function $u:[t, T] \times \Omega \times \mathbb{R} \rightarrow \mathbb{R}$ with $u(T, \cdot)=\xi$ a.e. is called decoupling field for $(\xi,(\mu, \sigma, f))$ on $[t, T]$ if for all $t_{1}, t_{2} \in[t, T]$ with $t_{1} \leq t_{2}$ and any $\mathcal{F}_{t_{1}}$-measurable $X_{t_{1}}: \Omega \rightarrow \mathbb{R}$ there exist progressively measurable processes $(X, Y, Z)$ on $\left[t_{1}, t_{2}\right]$ such that

$$
\begin{aligned}
X_{s} & =X_{t_{1}}+\int_{t_{1}}^{s} \mu\left(r, X_{r}, Y_{r}, Z_{r}\right) d r+\int_{t_{1}}^{s} \sigma\left(r, X_{r}, Y_{r}, Z_{r}\right) d W_{r}, \\
Y_{s} & =Y_{t_{2}}-\int_{s}^{t_{2}} f\left(r, X_{r}, Y_{r}, Z_{r}\right) d r-\int_{s}^{t_{2}} Z_{r} d W_{r}, \\
Y_{s} & =u\left(s, X_{s}\right),
\end{aligned}
$$

a.s. for all $s \in\left[t_{1}, t_{2}\right]$. In particular, we want all integrals to be well-defined.

Some remarks about this definition are in place.

- The first equation in (10) is called the forward equation, the second the backward equation and the third will be referred to as the decoupling condition.

- Note that, if $t_{2}=T$, we get $Y_{T}=\xi\left(X_{T}\right)$ a.s. as a consequence of the decoupling condition together with $u(T, \cdot)=\xi$. At the same time $Y_{T}=\xi\left(X_{T}\right)$, together with the decoupling condition, implies $u(T, \cdot)=\xi$ a.e.

- If $t_{2}=T$ we can say that a triplet $(X, Y, Z)$ solves the FBSDE, meaning that it satisfies the forward and the backward equation, together with $Y_{T}=\xi\left(X_{T}\right)$. This relationship $Y_{T}=\xi\left(X_{T}\right)$ is referred to as the terminal condition.

For the following we need to introduce further notation.

Let $I \subseteq[0, T]$ be an interval and $u: I \times \Omega \times \mathbb{R} \rightarrow \mathbb{R}$ a map such that $u(s, \cdot)$ is measurable for every $s \in I$. We define

$$
L_{u, x}:=\sup _{s \in I} \inf \left\{L \geq 0 \mid \text { for a.a. } \omega \in \Omega:\left|u(s, \omega, x)-u\left(s, \omega, x^{\prime}\right)\right| \leq L\left|x-x^{\prime}\right| \text { for all } x, x^{\prime} \in \mathbb{R}\right\},
$$

where $\inf \emptyset:=\infty$. We also set $L_{u, x}:=\infty$ if $u(s, \cdot)$ is not measurable for every $s \in I$. One can show that $L_{u, x}<\infty$ is equivalent to $u$ having a modification which is truly Lipschitz continuous in $x \in \mathbb{R}$.

We denote by $L_{\sigma, z}$ the Lipschitz constant of $\sigma$ w.r.t. the dependence on the last component $z$. We set $L_{\sigma, z}=\infty$ if $\sigma$ is not Lipschitz continuous in $z$.

By $L_{\sigma, z}^{-1}=\frac{1}{L_{\sigma, z}}$ we mean $\frac{1}{L_{\sigma, z}}$ if $L_{\sigma, z}>0$ and $\infty$ otherwise.

For an integrable real valued random variable $F$ the expression $\mathbb{E}_{t}[F]$ refers to $\mathbb{E}\left[F \mid \mathcal{F}_{t}\right]$, while $\mathbb{E}_{t, \infty}[F]$ refers to ess $\sup \mathbb{E}\left[F \mid \mathcal{F}_{t}\right]$, which might be $\infty$, but is always well defined as the infimum of all constants $c \in[-\infty, \infty]$ such that $\mathbb{E}\left[F \mid \mathcal{F}_{t}\right] \leq c$ a.s. Additionally, we write $\|F\|_{\infty}$ for the essential supremum of $|F|$.

In practice it is important to have explicit knowledge about the regularity of $(X, Y, Z)$. For instance, it is important to know in which spaces the processes live, and how they react to changes in the initial value. 
Definition 3.2. Let $u:[t, T] \times \Omega \times \mathbb{R} \rightarrow \mathbb{R}$ be a decoupling field to $(\xi,(\mu, \sigma, f))$.

1. We say $u$ to be weakly regular if $L_{u, x}<L_{\sigma, z}^{-1}$ and $\sup _{s \in[t, T]}\|u(s, \cdot, 0)\|_{\infty}<\infty$.

2. A weakly regular decoupling field $u$ is called strongly regular if for all fixed $t_{1}, t_{2} \in[t, T]$, $t_{1} \leq t_{2}$, the processes $(X, Y, Z)$ arising in (10) are a.e unique and satisfy

$$
\sup _{s \in\left[t_{1}, t_{2}\right]} \mathbb{E}_{t_{1}, \infty}\left[\left|X_{s}\right|^{2}\right]+\sup _{s \in\left[t_{1}, t_{2}\right]} \mathbb{E}_{t_{1}, \infty}\left[\left|Y_{s}\right|^{2}\right]+\mathbb{E}_{t_{1}, \infty}\left[\int_{t_{1}}^{t_{2}}\left|Z_{s}\right|^{2} d s\right]<\infty
$$

for each constant initial value $X_{t_{1}}=x \in \mathbb{R}$. In addition they are required to be measurable as functions of $(x, s, \omega)$ and even weakly differentiable w.r.t. $x \in \mathbb{R}^{n}$ such that for every $s \in\left[t_{1}, t_{2}\right]$ the mappings $X_{s}$ and $Y_{s}$ are measurable functions of $(x, \omega)$ and even weakly differentiable w.r.t. $x$ such that

$$
\begin{aligned}
& \operatorname{ess~sup}_{x \in \mathbb{R}} \sup _{s \in\left[t_{1}, t_{2}\right]} \mathbb{E}_{t_{1}, \infty}\left[\left|\partial_{x} X_{s}\right|^{2}\right]<\infty, \\
& {\operatorname{ess} \sup _{x \in \mathbb{R}}} \sup _{s \in\left[t_{1}, t_{2}\right]} \mathbb{E}_{t_{1}, \infty}\left[\left|\partial_{x} Y_{s}\right|^{2}\right]<\infty, \\
& \operatorname{ess} \sup _{x \in \mathbb{R}} \mathbb{E}_{t_{1}, \infty}\left[\int_{t_{1}}^{t_{2}}\left|\partial_{x} Z_{s}\right|^{2} \mathrm{~d} s\right]<\infty .
\end{aligned}
$$

3. We say that a decoupling field on $[t, T]$ is strongly regular on a subinterval $\left[t_{1}, t_{2}\right] \subseteq[t, T]$ if $u$ restricted to $\left[t_{1}, t_{2}\right]$ is a strongly regular decoupling field for $\left(u\left(t_{2}, \cdot\right),(\mu, \sigma, f)\right)$.

Under suitable conditions a rich existence, uniqueness and regularity theory for decoupling fields can be developed.

Assumption (SLC): $(\xi,(\mu, \sigma, f))$ satisfies standard Lipschitz conditions (SLC) if

1. $(\mu, \sigma, f)$ are Lipschitz continuous in $(x, y, z)$ with Lipschitz constant $L$,

2. $\|(|\mu|+|f|+|\sigma|)(\cdot, \cdot, 0,0,0)\|_{\infty}<\infty$,

3. $\xi: \Omega \times \mathbb{R} \rightarrow \mathbb{R}$ is measurable such that $\|\xi(\cdot, 0)\|_{\infty}<\infty$ and $L_{\xi, x}<L_{\sigma, z}^{-1}$.

In order to have a notion of global existence we need the following definition:

Definition 3.3. We define the maximal interval $I_{\max } \subseteq[0, T]$ of the problem given by $(\xi,(\mu, \sigma, f))$ as the union of all intervals $[t, T] \subseteq[0, T]$, such that there exists a weakly regular decoupling field $u$ on $[t, T]$.

Note that the maximal interval might be open to the left. Also, let us remark that we define a decoupling field on such an interval as a mapping which is a decoupling field on every compact subinterval containing $T$. Similarly we can define weakly and strongly regular decoupling fields as mappings which restricted to an arbitrary compact subinterval containing $T$ are weakly (or strongly) regular decoupling fields in the sense of the definitions given above.

Finally, we have global existence and uniqueness on the maximal interval: 
Theorem 3.4 ([6], Theorem 5.1.11, Lemma 5.1.12 and Corollary 2.5.5). Let $(\xi,(\mu, \sigma, f))$ satisfy SLC. Then there exists a unique strongly regular decoupling field $u$ on $I_{\max }$. Furthermore, either $I_{\max }=[0, T]$ or $I_{\max }=\left(t_{\min }, T\right]$, where $0 \leq t_{\min }<T$. In the latter case we have

$$
\lim _{t \downarrow t_{\min }} L_{u(t, \cdot), x}=L_{\sigma, z}^{-1} .
$$

Moreover, for any $t \in I_{\max }$ and any initial condition $X_{t}=x \in \mathbb{R}$ there is a unique solution $(X, Y, Z)$ of the FBSDE on $[t, T]$ satisfying

$$
\sup _{s \in[t, T]} \mathbb{E}\left[\left|X_{s}\right|^{2}\right]+\sup _{s \in[t, T]} \mathbb{E}\left[\left|Y_{s}\right|^{2}\right]+\mathbb{E}\left[\int_{t}^{T}\left|Z_{s}\right|^{2} d s\right]<\infty .
$$

Equality (13) allows to verify global existence, i.e. $I_{\max }=[0, T]$, via contradiction. We refer to this approach as the method of decoupling fields.

\section{Transforming the FBSDE}

The aim of this section is to prove that the adjoint FBSDE (6) has a solution on the whole interval $[0, T]$. To this end we first transform the FBSDE in a way that reduces the dependence of the forward diffusion on the control. We then apply the method of decoupling fields to the transformed system.

Let $\gamma=\frac{1}{\delta_{u}}$. We consider the auxiliary FBSDE

$$
\begin{aligned}
X_{s}^{x}= & x+\int_{0}^{s}\left(b_{r}+B_{r} M_{r}^{x}-B_{r}\left(X_{r}^{x}-M_{r}^{x}\right)-\gamma f_{m}\left(r, M_{r}^{x}, \tilde{Z}_{r}^{x}\right)\right) d r \\
& +\int_{0}^{s}\left(\tilde{Z}_{r}^{x}-\gamma f_{a}\left(r, M_{r}^{x}, \tilde{Z}_{r}^{x}\right)\right) d W_{r} \\
M_{s}^{x}= & \left(I d+\gamma g^{\prime}\right)^{-1}\left(X_{T}^{x}\right)-\int_{s}^{T} \tilde{Z}_{r}^{x} d W_{r}-\int_{s}^{T}\left(b_{r}+B_{r} M_{r}^{x}\right) \mathrm{d} r,
\end{aligned}
$$

for all $s \in[0, T]$.

We first show that the parameters of the FBSDE (14) satisfy the standard Lipschitz conditions.

Notice that $\gamma g^{\prime}$ is non-decreasing in $x$ since $g$ is convex. Hence $I d+\gamma g^{\prime}$ is strictly increasing with a derivative of at least 1 . This implies that the inverse in $x$, denoted by $\xi=\left(I d+\gamma g^{\prime}\right)^{-1}$, exists and that the inverse is Lipschitz continuous in $x$ with a Lipschitz constant smaller than or equal to 1 . Since $g^{\prime}(0)$ is essentially bounded, also $\xi(0)=\left(I d+\gamma g^{\prime}\right)^{-1}(0)$ is essentially bounded. Indeed,

$$
|\xi(0)|=\left|\xi(0)-\xi\left(\left(I d+\gamma g^{\prime}\right)(0)\right)\right| \leq\left|\left(I d+\gamma g^{\prime}\right)(0)\right| \leq \gamma\left\|g^{\prime}(0)\right\|_{\infty} .
$$

Let $\mu(t, x, m, z)=\left(b_{t}+B_{t} m\right)-B_{t}(x-m)-\gamma f_{m}(t, m, z)$ and $\sigma(t, m, z)=z-\gamma f_{a}(t, m, z)$ be the drift and the diffusion coefficient of the forward equation in (14), respectively. Condition (C1) entails that $\mu$ and $\sigma$ are Lipschitz continuous and that $(|\sigma|+|\mu|)(\cdot, \cdot, 0,0)$ is bounded.

Notice that $\sigma(t, m, z)$ is differentiable in $z$ and that the derivative takes values only in $\left[0,1-\frac{\delta_{l}}{\delta_{u}}\right]$. Hence the diffusion coefficient is Lipschitz continuous in $z$ with a Lipschitz constant equal to $\frac{\delta_{u}-\delta_{l}}{\delta_{u}}$. In particular, the Lipschitz constant of $\left(I d+\gamma g^{\prime}\right)^{-1}$ is strictly smaller than $L_{\sigma, z}^{-1}=\frac{\delta_{u}}{\delta_{u}-\delta_{l}}$. Note that $L_{\sigma, z}=0$ if and only if $\delta_{l}=\delta_{u}$.

To sum up, we have verified that the parameters of the FBSDE (14) satisfy the standard Lipschitz conditions (SLC). The benefit of considering (14) comes from the following observation. 
Lemma 4.1. If $\left(X_{t}, M_{t}, \tilde{Z}_{t}\right)$ is a solution of (14), then $\left(M_{t}, \frac{1}{\gamma}\left(X_{t}-M_{t}\right),-f_{a}\left(t, M_{t}, \tilde{Z}_{t}\right)\right)$ is a solution of $(6)$.

Proof. Let $\left(X_{t}, M_{t}, \tilde{Z}_{t}\right)$ be a solution of (14). In particular, all three processes are in $\mathcal{H}_{T}^{2}$. The linear growth condition on $f_{a}$ implies that also $\left(f_{a}\left(t, M_{t}, \tilde{Z}_{t}\right)\right)$ is in $\mathcal{H}_{T}^{2}$. Thus each process in the new triplet $\left(M_{t}, \frac{1}{\gamma}\left(X_{t}-M_{t}\right),-f_{a}\left(t, M_{t}, \tilde{Z}_{t}\right)\right)$ is in $\mathcal{H}_{T}^{2}$. A straightforward calculation shows that the three processes of the new triplet satisfy the dynamics (6).

We use the method of decoupling fields for proving that there exists a solution of (14) on $[0, T]$. Since the parameters of (14) satisfy the (SLC), there exists a maximal interval $I_{\max }$ with a weakly regular decoupling field $u$ (see Theorem 3.4).

In the following fix $t_{0} \in I_{\max }$. Let $(X, M, \tilde{Z})=\left(X^{t_{0}, x}, M^{t_{0}, x}, \tilde{Z}^{t_{0}, x}\right)$ be the solution of (14) on $\left[t_{0}, T\right]$ with initial value $x \in \mathbb{R}$ such that $M_{t}=u\left(t, X_{t}\right)$ a.s. for all $(t, x) \in\left[t_{0}, T\right] \times \mathbb{R}$.

According to strong regularity $u$ is weakly differentiable w.r.t. the initial value $x \in \mathbb{R}$. In the following we denote by $u_{x}$ a version of the weak derivative of $u$ w.r.t. $x$ such that it coincides with the classical derivative at all points for which it exists and with 0 everywhere else. Moreover, the processes $(X, M, \tilde{Z})$ are weakly differentiable w.r.t. $x$. We can formally differentiate the forward and the backward equation in (14). One can verify that one can interchange differentiation and integration and that a chain rule for weak derivatives applies (see Sections A.2 and A.3 in [6]). We thus obtain that for every version $\left(\partial_{x} X, \partial_{x} M, \partial_{x} \tilde{Z}\right)=$ $\left(\partial_{x} X^{t_{0}, x}, \partial_{x} M^{t_{0}, x}, \partial_{x} \tilde{Z}^{t_{0}, x}\right)$ of the weak derivative, such that for every $s \in\left[t_{0}, T\right]\left(\partial_{x} X_{s}, \partial_{x} M_{s}\right)$ is a weak derivative of $\left(X_{s}, M_{s}\right)$, we have for every $t \in\left[t_{0}, T\right]$ :

$$
\begin{aligned}
\partial_{x} X_{t}= & +\int_{t_{0}}^{t} B_{s}\left(2 \partial_{x} M_{s}-\partial_{x} X_{s}\right) \mathrm{d} s-\gamma\left(f_{m m}\left(s, M_{s}, \tilde{Z}_{s}\right) \partial_{x} M_{s}+f_{m a}\left(s, M_{s}, \tilde{Z}_{s}\right) \partial_{x} \tilde{Z}_{s}\right) d s \\
& +\int_{t_{0}}^{t}\left(\partial_{x} \tilde{Z}_{s}-\gamma f_{m a}\left(s, M_{s}, \tilde{Z}_{s}\right) \partial_{x} M_{s}-\gamma f_{a a}\left(s, M_{s}, \tilde{Z}_{s}\right) \partial_{x} \tilde{Z}_{s}\right) d W_{s}
\end{aligned}
$$

and

$$
\partial_{x} M_{t}=\xi^{\prime}\left(X_{T}\right) \partial_{x} X_{T}+\int_{t}^{T} B_{s} \partial_{x} M_{s} \mathrm{~d} s+\int_{t}^{T} \partial_{x} \tilde{Z}_{s} d W_{s}
$$

for $\mathbb{P} \otimes \lambda$ - almost all $(\omega, x) \in \Omega \times \mathbb{R}$.

By redefining $\left(\partial_{x} X, \partial_{x} M\right)$ as the right-hand-sides of (15) and (16) respectively, we obtain processes $\left(\partial_{x} X, \partial_{x} M\right)$ that are continuous in time for all $(\omega, x)$ but remain weak derivatives of $X, M$ w.r.t. $x$. From now on, we always assume that $\partial_{x} X$ and $\partial_{x} M$ are continuous in time. We also assume that for fixed $t \in\left[t_{0}, T\right]$ the mappings $\partial_{x} X_{t}$ and $\partial_{x} M_{t}$ are weak derivatives of $X_{t}$ and $M_{t}$ w.r.t. $x \in \mathbb{R}$. In particular $\partial_{x} X_{t_{0}}=1$ a.s. for almost all $x \in \mathbb{R}$.

In order to obtain bounds on the weak derivative $u_{x}$, we study the process $V_{t}:=u_{x}\left(t, X_{t}\right)$, $t \in\left[t_{0}, T\right]$.

Recall that $M_{t}=u\left(t, X_{t}\right)$ a.s. for all $(t, x) \in\left[t_{0}, T\right] \times \mathbb{R}$. Therefore, for fixed $t \in\left[t_{0}, T\right]$, the weak derivatives of the two sides of the equation w.r.t. $x \in \mathbb{R}$ must coincide up to a $\mathbb{P} \otimes \lambda$ - null set. The chain rule for weak derivatives (see Corollary 3.2 in [1] or Lemma A.3.1. in [6]) implies, for any fixed $t \in\left[t_{0}, T\right]$, that we have for $\mathbb{P} \otimes \lambda$ - almost all $(\omega, x)$

$$
\partial_{x} M_{t} \mathbf{1}_{\left\{\partial_{x} X_{t}>0\right\}}=u_{x}\left(t, X_{t}\right) \partial_{x} X_{t} \mathbf{1}_{\left\{\partial_{x} X_{t}>0\right\}}=V_{t} \partial_{x} X_{t} \mathbf{1}_{\left\{\partial_{x} X_{t}>0\right\}} .
$$

Now, choose a fixed $x \in \mathbb{R}$ such that $\partial_{x} X_{t_{0}}=1$ a.s., (17), (15), (16) are satisfied for almost all $(\omega, t) \in\left[t_{0}, T\right] \times \Omega$ and, in addition, (17) is satisfied for $t=t_{0}, \mathbb{P}$ - almost surely. Note 
that, since $\partial_{x} X, \partial_{x} M$ are continuous in time, (15) and (16) in fact hold for all $t \in\left[t_{0}, T\right], \mathbb{P}$ almost surely.

Observe that $V_{t}$ is bounded since $u_{x}(t, x)$ is bounded. Furthermore, according to the definition of the maximal interval, if $L_{\sigma, z}>0$, then there exists $\varepsilon>0$ such that for all $(t, x) \in\left[t_{0}, T\right] \times \mathbb{R}$ we have $\left|u_{x}(t, x)\right| \leq(1-\varepsilon) \frac{\delta_{u}}{\delta_{u}-\delta_{l}}$. A priori, $\varepsilon$ depends on $t_{0}$. We will see below that it can be chosen independently of $t_{0}$.

In the case $L_{\sigma, z}=0$ there exists a constant $K$ such that for all $(t, x) \in\left[t_{0}, T\right] \times \mathbb{R}$ we have $\left|u_{x}(t, x)\right| \leq K$. We will show below that $K$ can be chosen independently of $t_{0}$.

We now turn to the dynamics of $V$.

Lemma 4.2. The process $\left(V_{t}\right)_{t \in\left[t_{0}, T\right]}$ has a time-continuous version which is an Itô process. Moreover, there exists $\widehat{Z} \in \mathcal{H}_{t_{0}, T}^{2}$ such that $(V, \widehat{Z})$ is the unique solution of the BSDE

$$
V_{t}=\xi^{\prime}\left(X_{T}\right)-\int_{t}^{T} \widehat{Z}_{s} d W_{s}-\int_{t}^{T} \rho\left(s, V_{s}, \widehat{Z}_{s}\right) d s
$$

where $\rho: \Omega \times\left[t_{0}, T\right] \times \mathbb{R} \times \mathbb{R} \rightarrow \mathbb{R}$ is defined by

$$
\begin{aligned}
\rho(t, v, z)= & v\left[2 B_{t}(1-v)+\gamma f_{m m}\left(t, M_{t}, \tilde{Z}_{t}\right) v+\gamma f_{m a}\left(t, M_{t}, \tilde{Z}_{t}\right) h(t, v, z)\right] \\
& -z\left[-\gamma f_{m a}\left(t, M_{t}, \tilde{Z}_{t}\right) v+\left(1-\gamma f_{a a}\left(t, M_{t}, \tilde{Z}_{t}\right)\right) h(t, v, z)\right],
\end{aligned}
$$

with

$$
h(t, v, z)=\frac{z-\gamma f_{m a}\left(t, M_{t}, \tilde{Z}_{t}\right) v^{2}}{1-v\left[1-\gamma f_{a a}\left(t, M_{t}, \tilde{Z}_{t}\right)\right]} .
$$

Furthermore, $\widehat{Z} \in B M O(\mathbb{P})$, i.e. $\sup _{t \in\left[t_{0}, T\right]}\left\|\mathbb{E}\left[\int_{t}^{T}\left|\widehat{Z}_{s}\right|^{2} \mathrm{~d} s \mid \mathcal{F}_{t}\right]\right\|_{\infty}<\infty$.

Proof. Let $\tau_{n}=T \wedge \inf \left\{t \geq t_{0}: \partial_{x} X_{t} \leq \frac{1}{n}\right\}$. On $\left[t_{0}, \tau_{n}\right]$ we have $V_{t}=\partial_{x} M_{t} \frac{1}{\partial_{x} X_{t}}$, a.e. Hence $V$ has a version which is an Itô process on $\left[t_{0}, \tau_{n}\right]$. We denote the Itô process decomposition by

$$
V_{t}=u_{x}\left(t_{0}, x\right)+\int_{t_{0}}^{t} \widehat{Z}_{s} d W_{s}+\int_{t_{0}}^{t} \kappa_{s} d s, \quad t \in\left[t_{0}, \tau_{n}\right] .
$$

The product formula yields, on $\left[t_{0}, \tau_{n}\right]$,

$$
\begin{aligned}
d\left(V_{t} \partial_{x} X_{t}\right)= & V_{t}\left(B_{t}\left(2 \partial_{x} M_{t}-\partial_{x} X_{t}\right)-\gamma\left(f_{m m}\left(t, M_{t}, \tilde{Z}_{t}\right) \partial_{x} M_{t}+f_{m a}\left(t, M_{t}, \tilde{Z}_{t}\right) \partial_{x} \tilde{Z}_{t}\right)\right) d t \\
& +\partial_{x} X_{t} \kappa_{t} d t+\widehat{Z}_{t}\left(\partial_{x} \tilde{Z}_{t}-\gamma f_{m a}\left(t, M_{t}, \tilde{Z}_{t}\right) \partial_{x} M_{t}-\gamma f_{a a}\left(t, M_{t}, \tilde{Z}_{t}\right) \partial_{x} \tilde{Z}_{t}\right) d t \\
& +\left(\partial_{x} X_{t} \widehat{Z}_{t}+V_{t}\left[\partial_{x} \tilde{Z}_{t}-\gamma f_{m a}\left(t, M_{t}, \tilde{Z}_{t}\right) \partial_{x} M_{t}-\gamma f_{a a}\left(t, M_{t}, \tilde{Z}_{t}\right) \partial_{x} \tilde{Z}_{t}\right]\right) d W_{t}
\end{aligned}
$$

The drift and diffusion coefficients coincide with the coefficients in (16). This implies, using straightforward transformations, the definition of $h$ and the property $V_{t} \partial_{x} X_{t}=\partial_{x} M_{t}$,

$$
\partial_{x} \tilde{Z}_{t}=\partial_{x} X_{t} h\left(t, V_{t}, \widehat{Z}_{t}\right)
$$

and

$$
\begin{aligned}
\kappa_{t}= & \frac{1}{\partial_{x} X_{t}}\left[B_{t} \partial_{x} M_{t}\right. \\
& -V_{t}\left(B_{t}\left(2 \partial_{x} M_{t}-\partial_{x} X_{t}\right)-\gamma\left(f_{m m}\left(t, M_{t}, \tilde{Z}_{t}\right) \partial_{x} M_{t}+f_{m a}\left(t, M_{t}, \tilde{Z}_{t}\right) \partial_{x} \tilde{Z}_{t}\right)\right) \\
& \left.-\widehat{Z}_{t}\left(\partial_{x} \tilde{Z}_{t}-\gamma f_{m a}\left(t, M_{t}, \tilde{Z}_{t}\right) \partial_{x} M_{t}-\gamma f_{a a}\left(t, M_{t}, \tilde{Z}_{t}\right) \partial_{x} \tilde{Z}_{t}\right)\right] \\
= & \rho\left(t, V_{t}, \widehat{Z}_{t}\right) .
\end{aligned}
$$


We next show that the denominator of $h$ is bounded away from zero. Assume first $L_{\sigma, z}>0$. Then for all $v$ with $|v| \leq(1-\varepsilon) \frac{\delta_{u}}{\delta_{u}-\delta_{l}}$ we have $\left|v\left(1-\gamma f_{a a}\left(s, M_{s}, \tilde{Z}_{s}\right)\right)\right| \leq|v| \frac{\delta_{u}-\delta_{l}}{\delta_{u}} \leq(1-\varepsilon)$, and hence the denominator of $h\left(t, V_{t}, \widehat{Z}_{t}\right)$ is greater than or equal to $\varepsilon$. In the case where $L_{\sigma, z}=0$, the denominator is equal to 1 .

It remains to show that $\tau:=\lim _{n \rightarrow \infty} \tau_{n}=T$ a.s. To this end note that $\partial_{x} X_{t}$ satisfies, on $\left[t_{0}, \tau\right)$, the linear SDE

$$
d \partial_{x} X_{t}=\alpha_{t} \partial_{x} X_{t} d t+\beta_{t} \partial_{x} X_{t} d W_{t}
$$

where

$$
\alpha_{t}:=B_{t}\left(2 V_{t}-1\right)-\gamma\left(f_{m m}\left(t, M_{t}, \tilde{Z}_{t}\right) V_{t}+f_{m a}\left(t, M_{t}, \tilde{Z}_{t}\right) h\left(t, V_{t}, \widehat{Z}_{t}\right)\right)
$$

and

$$
\beta_{t}:=h\left(t, V_{t}, \widehat{Z}_{t}\right)-\gamma f_{m a}\left(t, M_{t}, \tilde{Z}_{t}\right) V_{t}-\gamma f_{a a}\left(t, M_{t}, \tilde{Z}_{t}\right) h\left(t, V_{t}, \widehat{Z}_{t}\right) .
$$

Consequently,

$$
\partial_{x} X_{t \wedge \tau_{n}}=\exp \left(\int_{t_{0}}^{t \wedge \tau_{n}}\left(\alpha_{s}-\frac{1}{2} \beta_{s}^{2}\right) \mathrm{d} s+\int_{t_{0}}^{t \wedge \tau_{n}} \beta_{s} \mathrm{~d} W_{s}\right)
$$

Note that $\alpha_{t}$ and $\beta_{t}$ are both bounded by $C\left(1+\left|\widehat{Z}_{t}\right|\right)$ for some sufficiently large $C>0$. We claim that $\widehat{Z}$ has a bounded BMO-norm with a bound which does not depend on $n$. Indeed, the pair $(V, \widehat{Z})$ can be interpreted as a solution of a quadratic BSDE on $\left[0, \tau_{n}\right]$. Since $V$ is bounded, standard results imply that $\widehat{Z}$ has a bounded BMO-norm (see e.g. Theorem A.1.11. in [6] for details).

Now if $\left(\lim _{n \rightarrow \infty} \partial_{x} X_{\tau_{n}}\right)(\omega)=0$ for some $\omega$, then $\left(\int_{t_{0}}^{\tau}\left|\widehat{Z}_{t}\right|^{2} \mathrm{~d} t\right)(\omega)=\infty$ must hold for the same $\omega$. This, however, is false for almost all $\omega$, due to $\widehat{Z}$ being a BMO-process on $\left[t_{0}, \tau\right)$. In other words, the continuous process $\partial_{x} X$ does not reach 0 with probability 1 and, therefore, $\lim _{n \rightarrow \infty} \tau_{n}=T$ a.s.

In particular $V_{t}=\partial_{x} M_{t} \frac{1}{\partial_{x} X_{t}}$ a.e. and $V$ has a time-continuous version.

In the following we assume that $V$ refers to the time-continuous version of Lemma 4.2.

Lemma 4.3. There exists a $\mathbb{P}$-equivalent probability measure $Q$ and a $Q-B M W^{Q}$ such that $(V, \widehat{Z})$ is the unique solution of the BSDE

$$
\begin{aligned}
V_{t}= & \xi^{\prime}\left(X_{T}\right)-\int_{t}^{T} \widehat{Z}_{s} d W_{s}^{Q}- \\
& \int_{t}^{T}\left(2 B_{s} V_{s}\left(1-V_{s}\right)+\gamma f_{m m}\left(s, M_{s}, \tilde{Z}_{s}\right) V_{s}^{2}-\gamma^{2} \frac{f_{m a}^{2}\left(s, M_{s}, \tilde{Z}_{s}\right)}{1-V_{t}\left(1-\gamma f_{a a}\left(s, M_{s}, \tilde{Z}_{s}\right)\right)} V_{t}^{3}\right) d s .
\end{aligned}
$$

Proof. Note that

$$
\begin{aligned}
\rho(t, v, z)= & 2 B_{t} v(1-v)+\gamma f_{m m}\left(t, M_{t}, \tilde{Z}_{t}\right) v^{2}-\gamma^{2} \frac{f_{m a}^{2}\left(s, M_{s}, \tilde{Z}_{s}\right)}{1-v\left(1-\gamma f_{a a}\left(s, M_{s}, \tilde{Z}_{s}\right)\right)} v^{3} \\
& -z \psi(t, v, z),
\end{aligned}
$$

where

$$
\psi(t, v, z)=-\gamma v \frac{f_{m a}\left(s, M_{s}, \tilde{Z}_{s}\right)}{1-v\left(1-\gamma f_{a a}\left(s, M_{s}, \tilde{Z}_{s}\right)\right)}-\gamma f_{m a}\left(t, M_{t}, \tilde{Z}_{t}\right) v+\left(1-\gamma f_{a a}\left(t, M_{t}, \tilde{Z}_{t}\right)\right) h(t, v, z) .
$$


Since $V$ is bounded, there exists a constant $C>0$ such that $\left|\psi\left(t, V_{t}, \widehat{Z}_{t}\right)\right| \leq C\left(1+\left|\widehat{Z}_{t}\right|\right)$ for all $t \in\left[t_{0}, T\right]$. Consequently $\psi\left(t, V_{t}, \widehat{Z}_{t}\right) \in B M O(\mathbb{P})$. In particular there exists a probability measure $Q \sim \mathbb{P}$ such that

$$
\frac{\mathrm{d} Q}{\mathrm{~d} \mathbb{P}}=\exp \left(\int_{t_{0}}^{T} \psi\left(t, V_{t}, \widehat{Z}_{t}\right) \mathrm{d} W_{t}-\frac{1}{2} \int_{t_{0}}^{T} \psi^{2}\left(t, V_{t}, \widehat{Z}_{t}\right) \mathrm{d} t\right)
$$

By Girsanov's theorem $W_{t}^{Q}:=W_{t}-\int_{t_{0}}^{t} \psi\left(s, V_{s}, \widehat{Z}_{s}\right) \mathrm{d} s, t \in\left[t_{0}, T\right]$, is a Brownian motion w.r.t. $Q$. Finally, observe that $V$ satisfies (18).

Lemma 4.4. For all $t \in\left[t_{0}, T\right]$ we have a.s. $q \leq V_{t} \leq 1$, where

$$
q:=\frac{\exp \left(-T\left(2\|B\|_{\infty}+\gamma\left\|f_{m m}\right\|_{\infty}\right)\right)}{1+\gamma\left\|g^{\prime \prime}\right\|_{\infty}} \in(0,1) .
$$

Proof. Let $\alpha(s, y)=-2 B_{t} y(1-y)-\gamma f_{m m}\left(s, M_{s}, \tilde{Z}_{s}\right) y^{2}+\gamma^{2} \frac{f_{m a}^{2}\left(s, M_{s}, \tilde{Z}_{s}\right)}{1-y\left(1-\gamma f_{a a}\left(s, M_{s}, \tilde{Z}_{s}\right)\right)} y^{3}$ be the generator of the BSDE (18).

In the case $L_{\sigma, z}>0$ one can modify $\alpha$ to a Lipschitz continuous generator by replacing $y$ with

$$
\left(y \vee\left(-(1-\varepsilon) \frac{\delta_{u}}{\delta_{u}-\delta_{l}}\right)\right) \wedge(1-\varepsilon) \frac{\delta_{u}}{\delta_{u}-\delta_{l}} .
$$

Indeed, $(V, \widehat{Z})$ solves also the modified BSDE since $\left|V_{s}\right| \leq(1-\varepsilon) \frac{\delta_{u}}{\delta_{u}-\delta_{l}}$. Notice that for all $v$ with $|v| \leq(1-\varepsilon) \frac{\delta_{u}}{\delta_{u}-\delta_{l}}$ we have $\left|v\left(1-\gamma f_{a a}\left(s, M_{s}, \tilde{Z}_{s}\right)\right)\right| \leq|v| \frac{\delta_{u}-\delta_{l}}{\delta_{u}} \leq(1-\varepsilon)$, which further implies that

$$
v \mapsto \frac{1}{1-v\left(1-\gamma f_{a a}\left(s, M_{s}, \tilde{Z}_{s}\right)\right)}
$$

takes values only in $\left[1, \frac{1}{\varepsilon}\right]$ and is Lipschitz continuous.

In the case $L_{\sigma, z}=0$ the generator $\alpha$ becomes Lipschitz continuous by replacing $y$ with $(y \vee-K) \wedge K$.

Now consider the cut-off function $c(v)=((v \vee 0) \wedge 1)$ and set

$$
\begin{aligned}
\check{\alpha}(s, v)= & -2 B_{t} c(v)(1-c(v))-\gamma f_{m m}\left(s, M_{s}, \tilde{Z}_{s}\right) c(v)^{2} \\
& +\gamma^{2} \frac{f_{m a}^{2}\left(s, M_{s}, \tilde{Z}_{s}\right)}{1-c(v)\left(1-\gamma f_{a a}\left(s, M_{s}, \tilde{Z}_{s}\right)\right)} c(v)^{3} .
\end{aligned}
$$

Observe that

$$
\begin{aligned}
\check{\alpha}(s, v) & \leq-2 B_{t} c(v)(1-c(v))-\gamma f_{m m}\left(s, M_{s}, \tilde{Z}_{s}\right) c(v)^{2}+\gamma \frac{f_{m a}^{2}\left(s, M_{s}, \tilde{Z}_{s}\right)}{\left.f_{a a}\left(s, M_{s}, \tilde{Z}_{s}\right)\right)} c(v)^{2} \\
& \leq-2 B_{t} c(v)(1-c(v))-\gamma c(v)^{2} \frac{1}{f_{a a}\left(s, M_{s}, \tilde{Z}_{s}\right)} \operatorname{det}\left(D^{2} f\right)\left(s, M_{s}, \tilde{Z}_{s}\right) \\
& \leq-2 B_{t} c(v)(1-c(v)) .
\end{aligned}
$$

Let $(\check{V}, \check{Z})$ be the solution of the BSDE with parameters $\left(\xi^{\prime}\left(X_{T}\right), \check{\alpha}\right)$. The comparison theorem, applied to $(\check{V}, \check{Z})$ and the BSDE with parameters $\left(1,-2 B_{t} c(1-c)\right)$, implies $\check{V}_{t} \leq 1$. 
Next, we estimate $\check{V}$ from below. Notice that

$$
\check{\alpha}(s, v) \geq-2\|B\|_{\infty} c(v)(1-c(v))-\gamma\left\|f_{m m}\right\|_{\infty} c(v)^{2} \geq-\left(2\|B\|_{\infty}+\gamma\left\|f_{m m}\right\|_{\infty}\right) c(v) .
$$

Moreover, $\xi^{\prime}(x)=\frac{1}{1+\gamma g^{\prime \prime}(\xi(x))} \geq \frac{1}{1+\gamma\left\|g^{\prime \prime}\right\|_{\infty}}$. A comparison with the BSDE with parameters

$$
\left(\frac{1}{1+\gamma\left\|g^{\prime \prime}\right\|_{\infty}},-\left(2\|B\|_{\infty}+\gamma\left\|f_{m m}\right\|_{\infty}\right) c\right)
$$

yields $\check{V}_{t} \geq q$.

Notice that this further entails that $(\check{V}, \check{Z})$ is also a solution of the BSDE (18). Uniqueness implies that $\check{V}=V$.

Recall that the diffusion coefficient in the forward part of (14), given by $\sigma(t, m, z)=$ $z-\gamma f_{a}(t, m, z)$, is Lipschitz continuous in $z$ with Lipschitz constant $\frac{\delta_{u}-\delta_{l}}{\delta_{u}}$. Lemma 4.4 implies that $0<q \leq u_{x}\left(t_{0}, x\right)=V_{t_{0}} \leq 1<\frac{\delta_{u}}{\delta_{u}-\delta_{l}}$.

Note that we have chosen a version of $V$ such that $V_{t}=\frac{\partial_{x} M_{t}}{\partial_{x} X_{t}}$ for all $t \in\left[t_{0}, T\right]$. Moreover, for $t=t_{0}$, we have $V_{t_{0}}=\frac{\partial_{x} M_{t_{0}}}{1}=u_{x}\left(t_{0}, x\right)$, a.s.

Since $x$ was chosen arbitrarily outside a $\lambda$ - null set, we have that the $u_{x}\left(t_{0}, \cdot\right)$ is essentially bounded by 1 . Since the bound does not depend on $t_{0}$, by Theorem 3.4 it must hold that $I_{\max }=[0, T]$. Moreover, the following holds true:

Proposition 4.5. There exists a unique weakly regular decoupling field u for (14) on $[0, T]$. Moreover, for every $t \in[0, T]$ we have $q \leq u_{x}(t, x) \leq 1$ for almost all $(\omega, x) \in \Omega \times \mathbb{R}$.

We can now formulate the main result of the section.

Theorem 4.6. Let $m \in \mathbb{R}$. Then

1. for all $t \in[0, T]$ the function $u(t, \cdot)$ has a Lipschitz continuous inverse $u^{-1}(t, \cdot), P$-a.s.,

2. there exists a solution $(M, Y, Z)$ of (6),

3. the function $\nu(t, m):=\frac{1}{\gamma}\left(u^{-1}(t, m)-m\right)$ is a decoupling field for $(6)$, i.e. $Y_{t}=\nu\left(t, M_{t}\right)$ for all $t \in[0, T]$,

4. an optimal control of problem (4) is given by

$$
\alpha_{t}^{*}=\tilde{Z}_{t},
$$

where $\tilde{Z}$ is part of the solution triplet of (14) with inital value $x=u^{-1}(0, m)$.

Proof. Since $u_{x}(t, \cdot)$ is bounded away from zero and bounded from above by $1, u(t, \cdot): \mathbb{R} \rightarrow \mathbb{R}$ is a bijection. Therefore, for every $m \in \mathbb{R}$ there is an $x \in \mathbb{R}$ such that $M_{0}=u(0, x)=m$. For this $x \in \mathbb{R}$ there exists according to Theorem 3.4 a unique triplet $(X, M, \tilde{Z})$ such that

$$
\sup _{s \in[0, T]} \mathbb{E}\left[\left|X_{s}\right|^{2}\right]+\sup _{s \in[0, T]} \mathbb{E}\left[\left|M_{s}\right|^{2}\right]+\mathbb{E}\left[\int_{0}^{T}\left|\tilde{Z}_{s}\right|^{2} d s\right]<\infty
$$

and such that FBSDE (14) is satisfied. Finally, using Lemma 4.1 we obtain a solution to FBSDE (6).

A straightforward calculation shows that for all $t \in[0, T]$ we have $\nu\left(t, M_{t}\right)=Y_{t}$. Finally, using Proposition 2.1 we obtain that $\tilde{Z}_{t}=f_{a}^{-1}\left(t, M_{t},-Z_{t}\right)$ is an optimal control for problem (4). 


\section{$5 \quad$ An illustrating example}

In general one can not expect the FBSDE (6) to possess a solution in closed form. For some examples, however, one can calculate the process $V$ explicitly. This allows then to obtain a candidate for the decoupling field of FBSDE (14) and hence to derive a solution of (6) in closed form. In the following we illustrate such an explicit construction for a particular choice of $f$ and $g$.

Let $\bar{a}_{t}$ be a bounded and progressively measurable process, $l>0$ and $L \geq 0$. Suppose that $f(t, m, a)=l m^{2}+\left(a-\bar{a}_{t}\right)^{2}$. Furthermore, let $b=0, B \in \mathbb{R}$ and $g(m)=L m^{2}$. We can choose $\delta_{u}=\delta_{l}=2$, and hence $\gamma=\frac{1}{2}$.

In this example we interpret $M_{t}$ as the state of a particle in a medium with temperature $\alpha_{t}$ at time $t . \bar{a}_{t}$ is the natural temperature process. Any cooling or heating entails quadratic costs. Problem (4) corresponds to the aim of steering the particle as close as possible to zero while keeping the costs for a temperature control low.

Notice that $\xi(x)=\frac{x}{1+L}$ and $\xi^{\prime}(x)=\frac{1}{1+L}$. Moreover, the function $\rho$ defined in Lemma 4.2 is given by

$$
\rho(t, v)=2 B v+(l-2 B) v^{2} .
$$

Therefore, the process $\left(V_{t}\right)$ is the solution of the Riccati equation

$$
V^{\prime}(t)=2 B V(t)+(l-2 B) V^{2}(t), \quad V(T)=\frac{1}{1+L} .
$$

By solving the Riccati equation we obtain explicit expressions for $V_{t}$.

1. case: $B=0$.

$$
V_{t}=\frac{1}{l(T-t)+1+L}
$$

2. case: $B \neq 0$.

$$
V_{t}=\frac{e^{-2 B(T-t)}}{1+L+\frac{l-2 B}{2 B}\left(1-e^{-2 B(T-t)}\right)}
$$

Note that $V_{t}$ does not depend on the spatial variable $x$. In particular, the decoupling field $u$ of (14) is affine linear in $x$. This implies that $M$ is affine linear in $X$.

Note that in this example the FBSDE (14) takes the form

$$
\begin{aligned}
& X_{t}=x+\int_{0}^{t}\left((2 B-l) M_{s}-B X_{s}\right) d s+\int_{0}^{t} \bar{a}_{s} d W_{s}, \\
& M_{t}=\frac{X_{T}}{1+L}-\int_{t}^{T} \tilde{Z}_{s} d W_{s}-\int_{t}^{T} B M_{s} d s .
\end{aligned}
$$

Assuming $M_{t}=V_{t} X_{t}$, the drift of the forward equation is linear in $X$, which allows to solve the equation explicitly.

Proposition 5.1. The solution of (19) is given by

$$
\begin{aligned}
X_{t} & =\left(x+\int_{0}^{t} H_{s}^{-1} \bar{a}_{s} d W_{s}\right) H_{t}, \\
M_{t} & =V_{t} X_{t}, \\
\tilde{Z}_{t} & =\bar{a}_{t} V_{t},
\end{aligned}
$$


where $H_{t}=e^{\int_{0}^{t}\left[(2 B-l) V_{s}-B\right] d s}$. In particular, the decoupling field for (19) is given by $u(t, x)=$ $V_{t} x$. Moreover an optimal control of problem (4) is given by

$$
\alpha_{t}^{*}=\bar{a}_{t} V_{t}
$$

(note that the optimal control does not depend on the initial condition).

Proof. Let $X, M$ and $\tilde{Z}$ be defined as in Proposition 5.1. A straightforward calculation shows that $X$ satisfies the dynamics

$$
\begin{aligned}
d X_{t} & =\left[(2 B-l) V_{t}-B\right] X_{t} d t+\bar{a}_{t} d W_{t} \\
& =\left((2 B-l) M_{t}-B X_{t}\right) d t+\bar{a}_{t} d W_{t} .
\end{aligned}
$$

We now verify that $M$ satisfies the backward equation in (19). First notice that $M$ satisfies the terminal condition $M_{T}=\frac{X_{t}}{1+L}$. The product formula implies

$$
\begin{aligned}
d M_{t} & =V_{t} d X_{t}+X_{t} d V_{t} \\
& =V_{t}\left[(2 B-l) V_{t}-B\right] X_{t} d t+V_{t} \bar{a}_{t} d W_{t}+X_{t}\left(2 B V_{t}+(l-2 B) V_{t}^{2}\right) d t \\
& =B M_{t} d t+\tilde{Z}_{t} d W_{t} .
\end{aligned}
$$

Hence $(X, M, \tilde{Z})$ satisfies (19).

\section{Linking the decoupling field to the HJB equation}

In this section we try to explain the link between the FBSDE and the HJB approach for solving (4). Our arguments are mainly heuristic.

Let us assume that $b, B, f, g$ do not depend on $\omega$. Consider (6) and assume that there exists a differentiable function $w:[0, T] \times \mathbb{R} \rightarrow \mathbb{R}$ such that $w\left(t, M_{t}^{m}\right)=Y_{t}^{m}$ a.s. for all initial values $m \in \mathbb{R}$ and all $t \in[0, T]$. Assuming that $w$ is continuously differentiable in time and twice continuously differentiable in space, we can apply the Itô formula to $w\left(t, M_{t}\right)$ :

$$
\begin{aligned}
Y_{t}=w\left(t, M_{t}\right)= & w(0, m)+\int_{0}^{t} w_{t}\left(s, M_{s}\right) \mathrm{d} s+\int_{0}^{t} w_{m}\left(s, M_{s}\right) f_{3}^{*}\left(s, M_{s},-Z_{s}\right) \mathrm{d} W_{s} \\
& +\int_{0}^{t} w_{m}\left(s, M_{s}\right)\left(b_{s}+B_{s} M_{s}\right) \mathrm{d} s+\frac{1}{2} \int_{0}^{t} w_{m m}\left(s, M_{s}\right)\left(f_{3}^{*}\left(s, M_{s},-Z_{s}\right)\right)^{2} \mathrm{~d} s .
\end{aligned}
$$

At the same time the backward equation in (6) yields

$$
Y_{t}=Y_{0}+\int_{0}^{t} Z_{s} \mathrm{~d} W_{s}-\int_{0}^{t}\left(B_{s} Y_{s}+f_{m}\left(s, M_{s}, f_{3}^{*}\left(s, M_{s},-Z_{s}\right)\right)\right) \mathrm{d} s .
$$

Comparing the martingale and the finite variation parts leads to

$$
\begin{gathered}
Z_{s}=w_{m}\left(s, M_{s}\right) f_{3}^{*}\left(s, M_{s},-Z_{s}\right) \\
w_{t}\left(s, M_{s}\right)+w_{m}\left(s, M_{s}\right)\left(b_{s}+B_{s} M_{s}\right)+\frac{1}{2} w_{m m}\left(s, M_{s}\right)\left(f_{3}^{*}\left(s, M_{s},-Z_{s}\right)\right)^{2} \\
=-\left(B_{s} w\left(s, M_{s}\right)+f_{m}\left(s, M_{s}, f_{3}^{*}\left(s, M_{s},-Z_{s}\right)\right)\right) .
\end{gathered}
$$


The first equation is equivalent to $w_{m}\left(s, M_{s}\right) \alpha_{s}+f_{a}\left(s, M_{s}, \alpha_{s}\right)=0$, where $\alpha_{s}:=f_{3}^{*}\left(s, M_{s},-Z_{s}\right)$. Assume that $w_{m}\left(s, M_{s}\right) \geq 0$ and let $\varphi:[0, T] \times \mathbb{R} \times[0, \infty) \rightarrow \mathbb{R}$ be the function such that $\alpha_{s}=\varphi\left(s, M_{s}, w_{m}\left(s, M_{s}\right)\right)$. Then we obtain

$$
\begin{gathered}
w_{t}\left(s, M_{s}\right)+w_{m}\left(s, M_{s}\right)\left(b_{s}+B_{s} M_{s}\right)+\frac{1}{2} w_{m m}\left(s, M_{s}\right)\left(\varphi\left(s, M_{s}, w_{m}\left(s, M_{s}\right)\right)\right)^{2} \\
=-B_{s} w\left(s, M_{s}\right)-f_{m}\left(s, M_{s}, \varphi\left(s, M_{s}, w_{m}\left(s, M_{s}\right)\right)\right) .
\end{gathered}
$$

Thus, it is natural to expect $w$ to satisfy the PDE

$$
\begin{gathered}
w_{t}(s, m)+w_{m}(s, m)\left(b_{s}+B_{s} m\right)+\frac{1}{2} w_{m m}(s, m)\left(\varphi\left(s, m, w_{m}(s, m)\right)\right)^{2} \\
=-B_{s} w(s, m)-f_{m}\left(s, m, \varphi\left(s, m, w_{m}(s, m)\right)\right) .
\end{gathered}
$$

Now let us look at the classical HJB approach to problem (4): The associated HJB equation for the value function $v:[0, T] \times \mathbb{R} \rightarrow \mathbb{R}$ has the form

$$
v_{t}(s, m)+\left(b_{s}+B_{s} m\right) v_{m}(s, m)-\sup _{a \in \mathbb{R}}\left[-\frac{1}{2} a^{2} v_{m m}(s, m)-f(s, m, a)\right]=0 .
$$

Assuming $v_{m m} \geq 0$, the optimal $a$ in the above expression is given by $\varphi\left(s, m, v_{m m}(s, m)\right)$, which is straightforward to verify using Fermat's theorem. We can rewrite the HJB equation as

$$
\begin{gathered}
v_{t}(s, m)+\left(b_{s}+B_{s} m\right) v_{m}(s, m)+\frac{1}{2} v_{m m}(s, m)\left(\varphi\left(s, m, v_{m m}(s, m)\right)\right)^{2} \\
=-f\left(s, m, \varphi\left(s, m, v_{m m}(s, m)\right)\right) .
\end{gathered}
$$

Note that $w(T, \cdot)=g^{\prime}$ and $v(T, \cdot)=g$. It is, therefore, natural to conjecture that $w=v_{m}$ holds true. We verify this by assuming that $v$ is three times continuously differentiable and by differentiating the HJB equation for $v$ w.r.t. $m$, thus obtaining an equation for $v_{m}=: \bar{w}$ :

$$
\begin{aligned}
& \bar{w}_{t}(s, m)+\bar{w}_{m}(s, m)\left(b_{s}+B_{s} m\right)+\frac{1}{2} \bar{w}_{m m}(s, m)\left(\varphi\left(s, m, \bar{w}_{m}(s, m)\right)\right)^{2}+B_{s} \bar{w}(s, m) \\
& +\bar{w}_{m}(s, m) \varphi\left(s, m, \bar{w}_{m}(s, m)\right)\left(\varphi_{m}\left(s, m, \bar{w}_{m}(s, m)\right)+\varphi_{3}\left(s, m, \bar{w}_{m}(s, m)\right) \bar{w}_{m m}(s, m)\right) \\
= & -f_{m}\left(s, m, \varphi\left(s, m, \bar{w}_{m}(s, m)\right)\right) \\
& -f_{a}\left(s, m, \varphi\left(s, m, \bar{w}_{m}(s, m)\right)\right)\left(\varphi_{m}\left(s, m, \bar{w}_{m}(s, m)\right)+\varphi_{3}\left(s, m, \bar{w}_{m}(s, m)\right) \bar{w}_{m m}(s, m)\right),
\end{aligned}
$$

where $\varphi_{3}$ is the derivative w.r.t. the last component. Due to the definition of $\varphi$ the above simplifies to

$$
\begin{gathered}
\bar{w}_{t}(s, m)+\bar{w}_{m}(s, m)\left(b_{s}+B_{s} m\right)+\frac{1}{2} \bar{w}_{m m}(s, m)\left(\varphi\left(s, m, \bar{w}_{m}(s, m)\right)\right)^{2} \\
=-B_{s} \bar{w}(s, m)-f_{m}\left(s, m, \varphi\left(s, m, \bar{w}_{m}(s, m)\right)\right) .
\end{gathered}
$$

Indeed, $w$ and $\bar{w}$ satisfy the same PDE, which makes it plausible to believe that it is in fact the same object.

Now note that while $v$ satisfies a fully non-linear PDE, the PDE satisfied by its spatial derivative $w$ is quasi-linear and hence is easier to analyze. In particular, the quasi-linearity of $w$ allows to reduce the problem to the FBSDE (6). Although the FBSDE is strongly coupled, it still crucially simplifies the problem. Indeed, as we have seen in Section 4, the method of decoupling fields allows to prove existence, uniqueness and regularity. 


\section{References}

[1] L. Ambrosio and G. Dal Maso. A general chain rule for distributional derivatives. Proc. Am. Math. Soc., 108(3):691-702, 1990.

[2] S. Ankirchner, C. Blanchet-Scalliet, and M. Jeanblanc. Controlling the Occupation Time of an Exponential Martingale. Appl. Math. Optim., 76(2):415-428, 2017.

[3] S. Ankirchner, A. Fromm, T. Kruse, and A. Popier. Optimal position targeting via decoupling fields. working paper or preprint, Apr. 2017.

[4] F. Da Lio and O. Ley. Convex Hamilton-Jacobi equations under superlinear growth conditions on data. Appl. Math. Optim., 63(3):309-339, 2011.

[5] F. Delarue. On the existence and uniqueness of solutions to FBSDEs in a non-degenerate case. Stochastic Process. Appl., 99(2):209-286, 2002.

[6] A. Fromm. Theory and applications of decoupling fields for forward-backward stochastic differential equations. PhD thesis, Humboldt-Universität zu Berlin, 2015.

[7] A. Fromm and P. Imkeller. Existence, Uniqueness and Regularity of Decoupling Fields to Multidimensional Fully Coupled FBSDEs. Preprint arXiv:1310.0499, 2013.

[8] A. Fromm, P. Imkeller, and D. Prömel. An FBSDE approach to the Skorokhod embedding problem for Gaussian processes with non-linear drift. Electron. J. Probab., 20:38 pp., 2015.

[9] I. Karatzas. Adaptive control of a diffusion to a goal and a parabolic Monge-Ampère-type equation. Asian J. Math., 1(2):295-313, 1997.

[10] I. Karatzas and W. D. Sudderth. Control and stopping of a diffusion process on an interval. Ann. Appl. Probab., 9(1):188-196, 1999.

[11] J. Ma, P. Protter, and J. Yong. Solving forward-backward stochastic differential equations explicitly - a four step scheme. Probab. Theory Relat. Fields, 98(3):339-359, 1994.

[12] J. Ma, Z. Wu, D. Zhang, and J. Zhang. On well-posedness of forward-backward SDEs - a unified approach. Ann. Appl. Probab., 25(4):2168-2214, 2015.

[13] J. Ma, H. Yin, and J. Zhang. On non-Markovian forward-backward SDEs and backward stochastic PDEs. Stochastic Process. Appl., 122(12):3980-4004, 2012.

[14] J. Ma and J. Yong. Forward-backward stochastic differential equations and their applications, volume 1702 of Lecture Notes in Mathematics. Springer-Verlag, Berlin, 1999.

[15] J. M. McNamara. Optimal control of the diffusion coefficient of a simple diffusion process. Math. Oper. Res., 8(3):373-380, 1983.

[16] J. M. McNamara, A. I. Houston, and E. J. Collins. Optimality models in behavioral biology. SIAM Rev., 43(3):413-466, 2001.

[17] E. Pardoux and S. Tang. Forward-backward stochastic differential equations and quasilinear parabolic PDEs. Probab. Theory Relat. Fields, 114(2):123-150, 1999. 
[18] S. Peng and Z. Wu. Fully coupled forward-backward stochastic differential equations and applications to optimal control. SIAM J. Control Optim., 37(3):825-843, 1999.

[19] V. C. Pestien and W. D. Sudderth. Continuous-time red and black: how to control a diffusion to a goal. Math. Oper. Res., 10(4):599-611, 1985.

[20] L. C. G. Rogers and D. Williams. Diffusions, Markov processes, and martingales. Vol. 2. Cambridge Mathematical Library. Cambridge University Press, Cambridge, 2000. Itô calculus, Reprint of the second (1994) edition.

[21] H. M. Soner, N. Touzi, and J. Zhang. Wellposedness of second order backward sdes. Probability Theory and Related Fields, 153(1):149-190, Jun 2012.

[22] J. Yong and X. Y. Zhou. Stochastic controls, volume 43 of Applications of Mathematics (New York). Springer-Verlag, New York, 1999. Hamiltonian systems and HJB equations. 